\title{
The Effects of Training to Reduce Automatic Action Tendencies Toward Alcohol Using the Virtual Alcohol Approach-Avoidance Task in Heavy Social Drinkers
}

\author{
Deok-Yong Kim, MA and Jang-Han Lee, PhD
}

\begin{abstract}
This study aimed to reduce the approach tendency toward alcohol among heavy social drinkers using the Virtual Alcohol Approach-Avoidance Training Task training. A total of 28 heavy social drinkers were randomly assigned to either the training group $(n=14)$ or the control group $(n=14)$. The training group was implicitly trained to avoid situations that involved drinking alcohol and to approach situations that involved drinking nonalcoholic beverages. On the other hand, the control group received a sham training condition with the same ratio of approach or avoidance of drinking either alcohol or a nonalcoholic beverage. All participants made three visits in a period of 2-3 weeks to participate in either the training or sham training. As a result, the training group showed a decrease in implicit approach tendencies toward alcohol, but not in explicit craving for alcohol. In contrast, the control group showed an increase in both implicit approach tendencies and explicit craving toward alcohol. These results indicate that the virtual reality training to avoid alcohol-related stimuli or environments might reduce automatic action tendencies toward alcohol, while simply being exposed to alcohol-related stimuli or environments might increase craving for alcohol in the sham training group. Our findings also suggest that, including not only visual stimuli but also auditory stimuli in a virtual environment might be a tool for changing approach bias.
\end{abstract}

Keywords: approach-avoidance training, alcohol addiction, virtual environment

\section{Introduction}

I

$\mathrm{N}$ INDIVIDUALS SUFFERING from alcohol misuse, such as

alcohol use disorder or heavy social drinking, craving and approach tendency toward alcohol increase when they are exposed to alcohol-related stimuli or environments. ${ }^{1}$ According to the incentive sensitization theory, repeated exposure to a potentially addictive substance heightens the value of the addictive stimuli or environment and reinforces the automatic approach action tendency toward addictive substances. $^{2}$ The dual process model of addiction predicts that addictive behavior is maintained because the automatic (i.e., impulsive or implicit) processes to approach rewards are faster than the deliberate (i.e., reflective or explicit) processes to control craving. ${ }^{3,4}$ One of the training to modify these automatic processes is the Approach-Avoidance Task training (AAT-training). ${ }^{5}$ The AAT-training might be an effective method to directly reduce automatic approach tendency toward alcohol. ${ }^{6}$

The AAT-training is a modification of the AAT, ${ }^{5}$ which measures the implicit, automatic approach tendency toward alcohol. $^{7}$ Contrary to the automatic tendency of persons with addiction to approach addictive substances, the AATtraining repeatedly conditions an avoidance response (e.g., pushing a joystick) to addictive stimuli (e.g., pictures of alcohol) and an approach response (e.g., pulling a joystick) to stimuli of the contrasting category (e.g., pictures of nonalcoholic beverages). Previous studies using picture stimuli demonstrated that after a single AAT-training session, alcohol avoidance bias increased and alcohol consumption decreased in undergraduates. ${ }^{5}$ In addition, the effectiveness of the training was also reported in alcohol-dependent patients, and the training predicted a lowering of the relapse rates. ${ }^{6}$ However, alcohol problem behaviors might be affected not only by alcoholic substances but also by environmental stimuli, such as social drinking situations. According to classical conditioning, the tendency to approach alcohol may be due to the association between alcohol-related stimuli and the environment. ${ }^{8,9}$ Therefore, to reduce the tendency to approach alcohol, counter-conditioning is necessary not only for alcohol-related stimuli but also for alcohol-related environments, including synesthetic stimuli. 
Virtual reality could provide stimuli from real-life situations, including a sense of multiple sense modalities, such as vision and hearing. ${ }^{10,11}$ According to previous studies, participants reported a higher subjective craving for addictive stimulus presented in virtual reality than for that presented as a picture. ${ }^{12,13}$ In the Virtual Approach-Avoidance Task (VAAT), which measures alcohol craving, heavy social drinkers showed more difficulty in avoidance of alcoholrelated situations than of nonalcohol-related situations. ${ }^{14}$ The VAAT could be more immersive in alcohol-related situations, leading to an increase in alcohol craving in social drinkers. Based on these advantages, this study aimed to train heavy social drinkers to modify their approach bias to alcohol by using the VAAT. We predicted that the Virtual Alcohol Approach-Avoidance Training Task (VAAATtraining) would reduce the level of alcohol craving among heavy social drinkers through repeated training to avoid drinking situations.

The aim of this study was to investigate whether the training effect of VAAAT-training reduces heavy social drinkers' automatic action tendencies toward alcohol. Specifically, we intended to evaluate the effectiveness of the AAT-training in virtual reality, which, unlike the use pictorial stimuli in previous studies, could produce a deeper sense of immersion. We hypothesized that both implicit approach bias toward alcohol and explicit craving among heavy social drinkers would decrease after the training tasks. On the other hand, in the sham training condition, no differences in implicit approach bias and explicit craving would be observed among them.

\section{Methods}

\section{Participants}

A total of 494 undergraduates completed the Alcohol Use Disorders Identification Test (AUDIT) ${ }^{15}$ and 28 of them $($ male $=16$, female $=12)$, who scored above 22 points on the AUDIT, were classified as heavy social drinkers. The participants were randomly assigned to either the training group $(n=14)$ or the control group $(n=14)$ in the order in which they were recruited, in consideration of gender. There were no differences in the AUDIT score and in demographic variables (age and gender) between the two groups. The severity of anxiety and impulsivity was also not different between the groups (Table 1).

\section{Materials}

\section{Alcohol Approach-Avoidance Implicit Association Test}

The Alcohol Approach-Avoidance Implicit Association Test (A-IAT) was used to measure an implicit approach tendency toward alcohol using differences in reaction time between the target and the attribute categories. The A-IAT consisted of two target categories (alcoholic drinks: Soju [Korean rice wine], whiskey, Chamisul soju [a type of a soju], alcohol, beer, draft beer; and nonalcoholic drinks: Coke, milk, Sprite, coffee, Fanta, juice) and two attribute categories (approach: near, intimate, closer, approach, contact, and sticky; and avoidance: unwilling, leave, withdraw, away, escape, and avoid). ${ }^{16}$ The A-IAT consisted of a total of seven blocks, with 40 trials in the third, fourth, sixth, and seventh blocks, and 20 trials in the other blocks. In the third,
Table 1. Mean (Standard Deviation)

FOR the Alcohol Use Disorders IDENTIFICATION Test

SCORE AND DEMOgRaphic Variables BetweEN the Training and the Control Group

\begin{tabular}{lll}
\hline & $\begin{array}{c}\text { Training group } \\
(\mathrm{m}=8, \mathrm{f}=6)\end{array}$ & $\begin{array}{c}\text { Control group } \\
(\mathrm{m}=9, \mathrm{f}=5)\end{array}$ \\
\hline Age & $22.36(2.31)$ & $22.79(2.89)$ \\
AUDIT & $24.07(1.49)$ & $24.93(3.15)$ \\
STAI-S & $46.29(9.22)$ & $42.57(11.10)$ \\
STAI-T & $48.14(8.81)$ & $44.50(9.67)$ \\
BAS & $36.36(4.29)$ & $37.71(4.36)$ \\
BIS & $18.57(4.50)$ & $17.14(3.53)$ \\
\hline
\end{tabular}

AUDIT, Alcohol Use Disorders Identification Test; BAS, behavioral approach system; BIS, behavioral inhibition system; STAI-S, State-Trait Anxiety Inventory-State; STAI-T: State-Trait Anxiety Inventory-Trait.

fourth, sixth, and seventh blocks, the target and attribute categories had to be categorized as quickly and correctly as possible. The A-IAT $D$-score was calculated using the difference in reaction time between the incongruent condition (the association between alcohol and avoidance category) and the congruent condition (the association between alcohol and approach category) in the third, fourth, sixth, and seventh blocks. A higher IAT $D$-score reflected stronger approach tendencies toward alcohol as it showed a stronger association between alcohol and approach tendency.

\section{Self-questionnaire}

The AUDIT, which consists of 10 items rated on a 4-point Likert scale, was used to measure the level of current alcohol use. This scale consists of three subcategories: alcohol intake, dependence, and adverse consequences (Cronbach's $\alpha=0.80$ ). ${ }^{15}$ The Alcohol Use Questionnaire (AUQ) was used to evaluate the urge to drink. The AUQ consists of eight items to be rated on a 7-point Likert scale (Cronbach's $\alpha=0.81){ }^{17}$ The State-Trait Anxiety Inventory-State and Trait (STAI-S, T), which consists of 20 items to be rated on a 4-point Likert, was used to evaluate the level of anxiety (STAI-S Cronbach's $\alpha=0.92$, STAI-T Cronbach's $\alpha=0.86$ ). ${ }^{18}$ The approach and avoidance tendencies were measured using the behavioral inhibition system/behavioral approach system (BIS/BAS) scale, consisting of 7 items for BIS and 13 items for BAS, each rated on a 4-point Likert scale (BIS Cronbach's $\alpha=0.76$, BAS Cronbach's $\alpha=0.67) .{ }^{19}$

\section{The Virtual Alcohol Approach-Avoidance Training Task}

The VAAAT-training was constructed by modifying the VAAT. ${ }^{14}$ The VAAAT-training was used to reduce the association between alcohol-related environmental stimuli and action tendencies, and consisted of 44 trials (4 practice trials and 40 training or sham training trials). In the virtual environment, the participants entered a building and moved along a hallway to meet two rooms. One of the two rooms had a scene related to social situations, while the other room was empty. If the participants entered the room with the social situation scene, they watched the virtual environmental situation, including the alcohol or nonalcohol scenes. The virtual environmental situations consisted of four alcoholrelated scenes (i.e., scenes of drinking beer with friends in 
a bar) and four nonalcohol-related scenes (i.e., scenes of drinking juice with friends in a cafe).

The participants were instructed to respond to color signals (red or green circle), which made them pull or push a joystick; the signals appeared immediately after the participants watched the scenes of the alcohol or nonalcohol-related situations during $\sim 5,000 \mathrm{~ms}$. In approach trials, the participants experienced an approach tendency with a zoom-in effect, when the joystick was pulled. In avoidance trials, the participants experienced an avoidance tendency with a zoom-out effect, when a joystick was pushed. The training group received avoidance of $90 \%$ from alcohol-related situations (18 trials) and $10 \%$ from nonalcohol-related situations (2 trials), approaching $90 \%$ to nonalcohol-related situations (18 trials) and $10 \%$ to alcohol-related situations ( 2 trials). On the other hand, the control group received the sham training in both alcohol- and nonalcohol-related situations with $50 \%$ avoidance condition (alcohol-avoidance condition: 10 trials and nonalcohol-avoidance condition: 10 trials) and 50\% approach condition (alcohol-approach condition: 10 trials and nonalcoholapproach condition: 10 trials). The sham training condition was designed with the same ratio of the approach and avoidance conditions so that the participants did not form an association between certain circumstances and action tendencies.

Each session lasted about an hour, and each trial took about 40-60 seconds, including moving time in the virtual environment, situation presentation, and reaction time. To avoid participant fatigue, the total duration of trials in one session did not exceed an hour. In addition, the participants performed on the following training trials at least 4-5 days after the training, and the training period lasted for 2-3 weeks according to the participant's schedule.

\section{Procedure}

The study was conducted in three sessions over 3 days. All participants were asked to visit the laboratory three times within 2-3 weeks to conduct the program sessions (at the interval of 4-5 days). Upon arrival at the laboratory, all participants signed an informed consent form for participating in the study. To reduce any demand effect, participants were led to believe that they were taking part in a study on cognitive evaluation in social drinking situations.

The participants were randomly assigned to either the training group or the control group. All participants were informed about the procedure and asked to complete the questionnaires such as that for demographic data and the AUQ. The training group was trained using the VAAATtraining to reduce the association between alcohol-related situations and the approach tendency, while the participants in the control group were allocated to the sham training (where no association between certain situations and action tendencies was assumed). Implicit and explicit alcohol approach tendencies were measured on the first day and the last day.

In the debriefing, the researcher explained the purpose of the study and the experimental procedure to the participants and paid 15,000 won (about $\$ 15$ ) as a reward for their participation in the experiment. If the participants reported severe psychological discomfort after the experiment, the researchers were contacted to guide them to take proper steps. All study procedures were approved by the university Institutional Review Board.

\section{Data analysis}

To study the effects of the training on the automatic approach tendency toward alcohol, a two-way repeatedmeasures ANOVA was conducted, with group (training and control) as a between-subject factor and time (pretraining and posttraining) as a within-subject factor. The dependent variables were the implicit measurement ( $D$-scores on the A-IAT) and the explicit measurement (the AUQ score). Paired $t$-test was performed to compare the differences between the pretraining and posttraining in both groups.

\section{Results}

\section{Implicit association between alcohol and approach tendency}

The interaction effect between group and time was significant $\left[F(1,26)=25.28, p<0.001, \eta_{p}{ }^{2}=0.49\right]$ (Fig. 1). Paired $t$-test was performed to investigate the difference between pretraining and posttraining in each group. In addition, the difference of A-IAT $D$-score between pretraining and posttraining was significant in both groups (training group: $t(13)=2.77, p<0.05$, Cohen's $d=0.42$; Control group: $t(13)=-4.38, p<0.01$, Cohen's $d=0.78)$. These results indicate that after active training using the VAAATtraining, there was a decrease in implicit approach toward alcohol. In contrast, after sham training, there was an increase in implicit approach toward alcohol in the control group (Fig. 1). However, the main effect of group $[F(1$, 26) $=0.01, p=0.92]$ and time $[F(1,26)=1.05, p=0.32]$ did not attain statistical significance. The A-IAT $D$-score between the groups differed neither in the pretraining $[t(26)=1.50, p=0.15]$ nor in the posttraining $[t(26)=-1.56$, $p=0.13]$.

\section{Explicit cravings toward alcohol}

The analysis of the explicit alcohol approach tendencies revealed that the interaction effect between group and time was significant $\left[F(1,26)=8.06, p<0.01, \eta_{p}{ }^{2}=0.24\right]$ (Fig. 2). However, the main effects of group $[F(1,26)=0.03, p=$ $0.86]$ and time $[F(1,26)=0.04, p=0.85]$ were not significant. To investigate the difference in the explicit craving toward alcohol pretraining and posttraining in each group, a

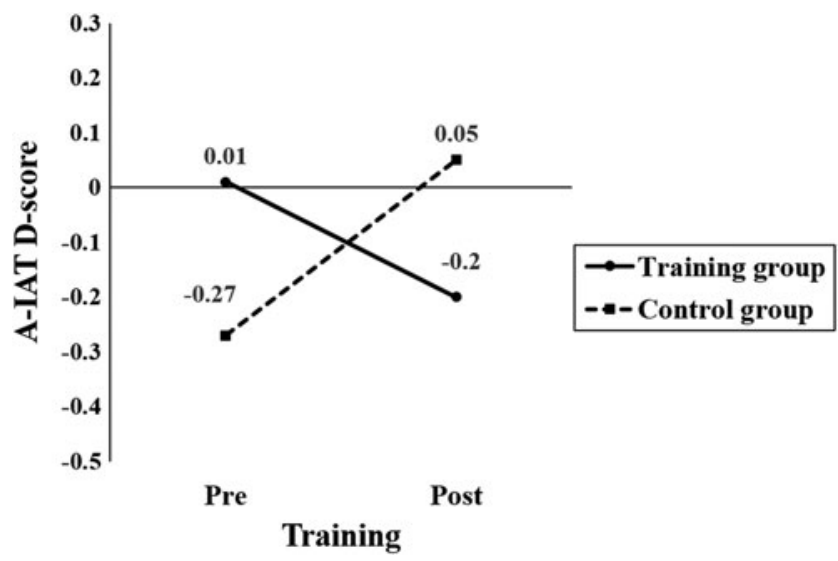

FIG. 1. The A-IAT $D$ score differences between training and control group. A-IAT, Alcohol Approach-Avoidance Implicit Association Test. 


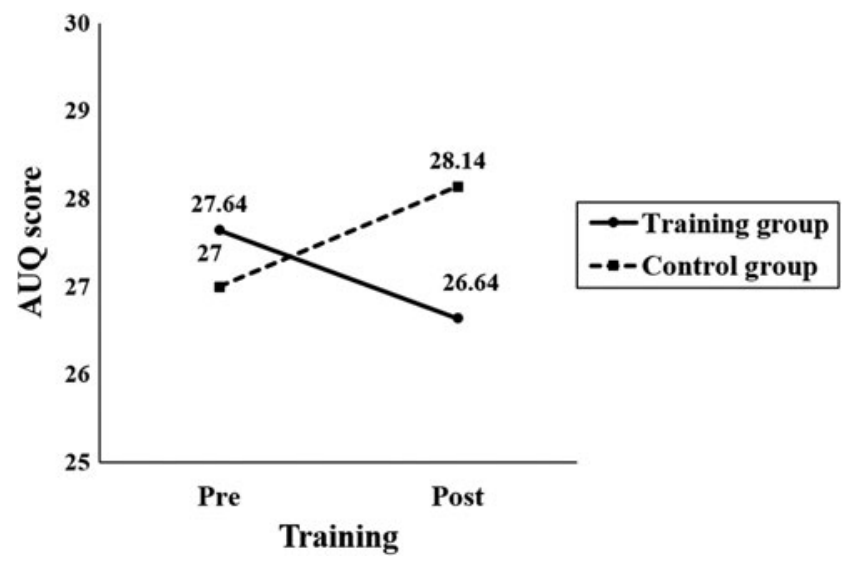

FIG. 2. The AUQ score differences between training and control group. AUQ, Alcohol Use Questionnaire.

followup test was performed. The results showed that, while the AUQ score was significantly different in the control group $[t(13)=-2.23, p<0.05$, Cohen's $d=0.17]$, it was not so in the training group $[t(13)=1.80, p=0.10]$. This suggests that the explicit craving toward alcohol after sham training increased in the control group (Fig. 2). On the other hand, the AUQ score between the groups differed neither in the pretraining $[t(26)=0.24, p=0.81]$ nor in the posttraining $[t(26)=-.64, p=0.53]$.

\section{Discussion}

This study aimed to investigate whether the VAAATtraining could reduce implicit action tendency and explicit craving toward alcohol in heavy social drinkers. Our main findings are as follows: after the VAAAT-training, the implicit approach bias toward alcohol decreased in the training group; however, the explicit craving for alcohol did not reduce significantly. This finding is consistent with previous studies that used AAT-training with pictures. ${ }^{5,6,20}$ These studies carried out AAT-training using pictures with 200600 trials each session, across $1-12$ weeks. ${ }^{21-23}$ However, in this study, the virtual environment produced significant training effects with 44 trials in each session, across 2-3 weeks. The effect size of the training in this study was medium (Cohen's $d=0.42$ ). These results indicate that virtual environment training could help modify action tendencies by providing more immersive experiences that contain both visual and auditory modalities.

However, the difference between pretraining and posttraining levels of explicit craving in the training group did not achieve statistical significance. One possible explanation for these findings is that the implicit and explicit craving might involve different processes. The VAAAT-training is focused on training to reduce implicit approach bias, but not explicit craving. According to the dual process model, explicit craving could be affected by more reflective and deliberate processes. ${ }^{4}$ Another possible explanation for these results is that the small sample or the number of trials could have contributed to a weaker effect. Both groups were randomly sampled from the population, with no differences in demographic and personality characteristics between both of them. Therefore, while our pool of participants was homogenous, a larger number of participants would be needed to analyze the potential effects. Furthermore, while the virtual environment provided more immersion than photographic stimuli, the small number of trials in the VAAAT-training might have limited the participants' fatigue and thus not induced explicit changes. Further research would be necessary to demonstrate the effects of the training better.

Unlike in the results of the training group, approach bias and explicit craving in the control group increased. The control group received sham training, which contained an equal number of avoidance and approach trials. We expected that the sham training would maintain the implicit approach bias and explicit craving; however, the results indicated an increase in both variables. However, the difference in presham training and postsham training AUQ scores in the control group had a small effect size (Cohen's $d=0.17$ ). This finding may be interpreted as the level of craving remaining unchanged. Another possible explanation is that the repeated exposure to alcohol-related situations increased the approach bias and cravings in the participants. In a previous study with undergraduates, the sham training group ate more unhealthy foods than healthy foods. ${ }^{24}$ The alcohol-related situations in the VAAAT-training contained realistic visual and auditory stimuli. Further research is necessary to investigate whether the level of craving would change due to repeated exposure to addictive stimuli in virtual reality.

In this study, the VAAAT-training was tested with heavy social drinkers. In further research, it would be necessary to verify its effectiveness on clinically diagnosed alcohol use disorder. Yet, the variables such as socioeconomic status and ethnicity might also play a part in these findings, so further studies are needed to take into account the relevant variables. In this study, we did not assess the amount or frequency of alcohol consumption in the participants before and after the training. Thus, followup studies need to be conducted to find out whether the VAAAT-training would be generalized to various drinking situations and lead to a reduction in actual alcohol consumption.

Despite the limitations outlined above, the results of this study demonstrate that the VAAAT-training is an effective training approach using a virtual environment with both visual and auditory stimuli. The VAAAT-training is also one of the training methods with ecological validation, as it allows for a direct counter conditioning for both addictive substances and the context or situations. ${ }^{25}$ In future studies, it is necessary to assess the validity of the VAAAT-training by comparing the effects observed in this study with AAT-training using pictorial stimuli, as well as with other interventions using virtual reality, such as cue exposure and aversion therapies.

\section{Author Disclosure Statement}

No competing financial interests exist.

\section{Funding Information}

This research was supported by the National Research Foundation of Korea Grant funded by the Korean Government (NRF-2013S1A5A2A01019443).

\section{References}

1. Field M, Kiernan A, Eastwood B, et al. Rapid approach responses to alcohol cues in heavy drinkers. Journal of 
Behavior Therapy and Experimental Psychiatry 2008; 39: 209-218.

2. Robinson TE, Berridge KC. The neural basis of drug craving: an incentive sensitization theory of addiction. Brain Research Reviews 1993; 18:247-291.

3. Strack F, Deutsch R. Reflective and impulsive determinants of social behavior 2004. Personality and Social Psychology Review 2004; 8:220-247.

4. Gladwin TE, Figner B, Crone EA, et al. Addiction, adolescence, and the integration of control and motivation. Developmental Cognitive Neuroscience 2011; 1:364-376.

5. Wiers RW, Rinck M, Kordts R, et al. Re-training automatic action-tendencies to approach alcohol in hazardous drinkers. Addiction 2010; 105:279-287.

6. Wiers RW, Eberl C, Rinck M, et al. Retraining automatic action tendencies changes alcoholic patients' approach bias for alcohol and improves treatment outcome. Psychological Science 2011; 22:490-497.

7. De Houwer J, Crombez G, Baeyens F, et al. On the generality of the affective Simon effect. Cognition and Emotion 2001; 15:189-206.

8. Bardo MT, Benvins RA. Conditioned place preference: what does it add to our preclinical understanding of drug reward? Psychopharmacology 2000; 153:31-43.

9. Weerts EM, Goodwin AK, Kaminski BJ, et al. Environmental cues, alcohol seeking, and consumption in Baboons: effects of response requirement and duration of alcohol abstinence. Alcoholism: Clinical and Experimental Research 2006; 30:2026-2036.

10. Ghiţă A, Gutiérrez-Maldonado J. Applications of virtual reality in individuals with alcohol misuse: a systematic review. Addictive Behaviors 2018; 81:1-11.

11. Riva G, Morganti F, Villamira M. Immersive virtual telepresence: virtual reality meets eHealth. Studies in Health Technology and Informatics 2004; 99:255-262.

12. Ferrer-Garcia M, Garcia-Rodriguez O, GutierrezMaldonado J, et al. Efficacy of virtual reality in triggering the craving to smoke: its relation to level of presence and nicotine dependence. Studies in Health Technology and Informatics 2010; 154:123-127.

13. García-Rodríguez O, Weidberg S, Gutiérrez-Maldonado J, et al. Smoking a virtual cigarette increase craving among smokers. Addictive Behaviors 2013; 38:2551-2554.

14. Kim D, Lee J. Development of a virtual approachavoidance task to assess alcohol cravings. Cyberpsychology, Behavior, and Social Networking 2015; 18:763-766.

15. Saunders JB, Aasland OG, Babor TF, et al. Development of the Alcohol Use Disorders Identification Test (AUDIT): WHO collaborative project on early detection of persons with harmful alcohol consumption-II. Addiction 1993; 88: 791-804.

16. Choi YJ, Lee J. The effects of virtual covert sensitization on reducing alcohol craving in heavy social drinkers. Virtual Reality 2015; 19:111-117.

17. Bohn MJ, Krahn DD, Staehler BA. Development and initial validation of measure of drinking urge in abstinent alcoholism. Alcoholism: Clinical and Experimental Research 1995; 19:600-606.

18. Spielberger CD, Gorsuch RI, Lushene RE. (1970) Manual for the State-Trait Anxiety Inventory. Palo Alto, CA: Consulting Psychologist Press.

19. Carver CS, White TL. Behavioral inhibition, behavioral activation, and affective responses to impending reward and punishment: the BIS/BAS scale. Journal of Personality and Social Psychology 1994; 67:319-333.

20. Wiers RW, Houben K, Fadardi JS, et al. Alcohol cognitive bias modification training for problem drinkers over the web. Addictive Behaviors 2015; 40:1-26.

21. Eberl C, Wiers RW, Pawelczack S, et al. Implementation of approach bias re-training in alcoholism-how many sessions are needed? Alcoholism: Clinical and Experimental Research 2014; 38:587-594.

22. Lindgren KP, Wiers RW, Teachman BA, et al. Attempted training of alcohol approach and drinking identity associations in US undergraduate drinkers: null results from two studies. PLoS One 2015; 10:e0134642.

23. Kakoschke N, Kemps E, Tiggemann M. Approach bias modification training and consumption: a review of the literature. Addictive Behaviors 2017; 64:21-28.

24. Becker D, Jostmann NB, Wiers RW, et al. Approach avoidance training in the eating domain: testing the effectiveness across three single session studies. Appetite 2015; 85:58-65.

25. Valmaggia LR, Latif L, Kempton MJ, et al. Virtual reality in the psychological treatment for mental health problems: an systematic review of recent evidence. Psychiatry Research 2016; 236:189-195.

\author{
Address correspondence to: \\ Prof. Jang-Han Lee \\ Department of Psychology \\ Chung-Ang University \\ 84 Heukseok-ro, Dongjak-gu \\ Seoul 06974 \\ Korea
}

E-mail: clipsy@cau.ac.kr 